\title{
Budesonide-Loaded Pectin/Polyacrylamide Hydrogel for Sustained Delivery: Fabrication, Characterization and In Vitro Release Kinetics
}

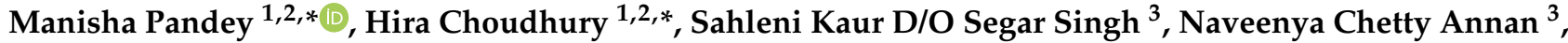 \\ Subrat Kumar Bhattamisra ${ }^{4}$, Bapi Gorain ${ }^{5,6}$ and Mohd Cairul Iqbal Mohd Amin ${ }^{7}$
}

check for updates

Citation: Pandey, M.; Choudhury, H.; D/O Segar Singh, S.K.; Chetty Annan, N.; Bhattamisra, S.K.; Gorain, B.; Mohd Amin, M.C.I. BudesonideLoaded Pectin/Polyacrylamide Hydrogel for Sustained Delivery: Fabrication, Characterization and In Vitro Release Kinetics. Molecules 2021, 26, 2704. https://doi.org/10.3390/ molecules26092704

Academic Editor: Ewelina Piktel

Received: 12 March 2021

Accepted: 13 April 2021

Published: 5 May 2021

Publisher's Note: MDPI stays neutral with regard to jurisdictional claims in published maps and institutional affiliations.

Copyright: (c) 2021 by the authors. Licensee MDPI, Basel, Switzerland. This article is an open access article distributed under the terms and conditions of the Creative Commons Attribution (CC BY) license (https:// creativecommons.org/licenses/by/ $4.0 /)$.
1 Department of Pharmaceutical Technology, School of Pharmacy, International Medical University, Bukit Jalil, Kuala Lumpur 57000, Malaysia

2 Centre for Bioactive Molecules and Drug Delivery, Institute for Research, Development and Innovation, International Medical University, Kuala Lumpur 57000, Malaysia

3 Bachelor of Pharmacy Student, School of Pharmacy, International Medical University, Kuala Lumpur 57000, Malaysia; sahlenikaur95@gmail.com (S.K.D.S.S.); naveenya_vee@yahoo.com (N.C.A.)

4 Department of Life sciences, School of Pharmacy, International Medical University-Bukit Jalil, Kuala Lumpur 57000, Malaysia; subratkumar@imu.edu.my

5 School of Pharmacy, Faculty of Health and Medical Sciences, Taylor's University, Subang Jaya 47500, Malaysia; bapi.gn@gmail.com

6 Center for Drug Delivery and Molecular Pharmacology, Faculty of Health and Medical Sciences, Taylor's University, Subang Jaya 47500, Malaysia

7 Centre for Drug Delivery Technology, Faculty of Pharmacy, Universiti Kebangsaan Malaysia, Jalan Raja Muda Abdul Aziz, Kuala Lumpur 50300, Malaysia; mciamin@ukm.edu.my

* Correspondence: manishapandey@imu.edu.my (M.P.); hirachoudhury@imu.edu.my (H.C.); Tel.: +60-166-048-589 (M.P.); +60-183-830-420 (H.C.)

Abstract: A single ulcerative colitis (UC) is a chronic inflammatory bowel disease (IBD) that causes inflammation of the colonic mucosa at the distal colon and rectum. The mainstay therapy involves anti-inflammatory immunosuppression based on the disease location and severity. The disadvantages of using systemic corticosteroids for UC treatment is the amplified risk of malignancies and infections. Therefore, topical treatments are safer as they have fewer systemic side effects due to less systemic exposure. In this context, $\mathrm{pH}$ sensitive and enzymatically triggered hydrogel of pectin (PC) and polyacrylamide (PAM) has been developed to facilitate colon-targeted delivery of budesonide (BUD) for the treatment of UC. The hydrogels were characterized by Fourier transform infrared spectroscopy (FTIR), X-ray diffraction (XRD), swelling ratio, and drug release. FT-IR spectroscopy confirmed the grafting as well loading of BUD in hydrogel. XRD showed the amorphous nature of hydrogel and increment in crystallinity after drug loading. On the other hand, SEM showed that the hydrogels exhibited a highly porous morphology, which is suitable for drug loading and also demonstrated a $\mathrm{pH}$-responsive swelling behaviour, with decreased swelling in acidic media. The in-vitro release of BUD from the hydrogel exhibited a sustained release behaviour with non-ficken diffusion mechanism. The model that fitted best for BUD released was the Higuchi kinetic model. It was concluded that enzyme/pH dual-sensitive hydrogels are an effective colon-targeted delivery system for UC.

Keywords: ulcerative colitis; hydrogel; pectin; colon drug delivery; $\mathrm{pH}$-sensitive

\section{Introduction}

Ulcerative colitis (UC) is a chronic inflammatory bowel disease (IBD) that causes inflammation of the colonic mucosa at the distal colon and rectum. Symptoms include bloody diarrhea, abdominal pain, tenesmus, and unexplained weight loss [1]. The mainstay therapy involves anti-inflammatory immunosuppression based on the disease location and severity. UC treatments comprise 5-aminosalicylic acid (5-ASA) drugs, corticosteroids, and immunosuppressants [2]. 
For a long time, the treatment options for mild to moderate UC were constricted to oral and rectal 5-ASA formulations as well as rectal corticosteroid preparations [3]. The disadvantage of using systemic corticosteroids for UC treatment is the amplified risk of malignancies and infections [4,5]. Topical treatments are safer as they have fewer systemic side effects due to lesser systemic exposure [5].

An alternative to prednisolone is a local corticosteroid, budesonide. Budesonide is related structurally to $16 \alpha$-hydroxyprednisolone and has anti-inflammatory properties [6]. Budesonide has a 15 times higher affinity for glucocorticoid receptors and better topical potency that is almost five times more than that of prednisone [7]. The drug is cleared rapidly through high first-pass metabolism in the liver, resulting in low systemic bioavailability of only about $10 \%[6,7]$. Due to this limited systemic exposure of budesonide, its systemic side effects are much lower than conventional corticosteroids. When compared against prednisolone and other corticosteroids at clinically equivalent doses, budesonide shows hypothalamic-pituitary-adrenal axis suppression and steroid-associated side effects that are significantly lower $[6,8]$. Studies show that the limitation of its low bioavailability can be overcome by developing a colon-targeted delivery system for budesonide [9].

In 2013, the oral extended-release formulation and rectal application of budesonide gained approval by the US Food and Drug Administration (FDA) for remission induction in patients with mild to moderate UC [10]. At present, there are three budesonide formulations, two of which are standard capsules both designed to release the drug only at the distal ileum and proximal colon, which are not UC target sites [11]. The latest commercially available formulation, the budesonide multimatrix (MMX) capsule, was designed in the year 2014 to release the drug throughout the entire colon [7,12]. This formulation has been shown to have significant remission induction in patients with UC [13].

Budenofalk $^{\circledR}$ and Entocort ${ }^{\circledR}$ are examples of a strategy developed by coating budesonide with a $\mathrm{pH}$-sensitive polymer $[13,14]$.

Similarly, polysaccharide-based delivery systems have been extensively studied and proven to be a promising colon-targeted delivery system [15-17]. Polysaccharides are abundantly available and can be easily modified both chemically and biochemically $[16,18]$. A unique characteristic of these polysaccharides is the ability to remain as a whole in the acidic nature of the stomach as well as in the environment of the small intestines. Conversely, polysaccharides are degraded by microflora that is found in the colon making it a desirable carrier system for polypeptide drugs used to treat colonic diseases, such as UC [19].

Ye Liu and Hong Zhou conducted a study in which a natural polysaccharide, guar gum was used to develop a budesonide colon-targeted system. This study investigated the invitro drug release in simulated gastric fluid ( $\mathrm{pH}$ 1.2) and simulated intestinal fluid ( $\mathrm{pH} 7.5)$. It revealed a slower, sustained release pattern of budesonide. This shows that budesonideloaded natural polysaccharide formulation was able to withstand the acidic nature of the stomach and intestinal environment due to the gel-forming ability of polysaccharides. A similar study was conducted by Krishnaiah et al. whereby a formulation of compression coated guar gum tablets was developed [20].

Since the colon is the target site for UC treatment, a colon-targeted hydrogel will be a good novel drug-delivery system for budesonide. Hydrogels are three-dimensional hydrophilic polymeric networks synthesized by physical and chemical crosslinking methods $[21,22]$.

In this project, $\mathrm{pH}$-sensitive enzyme-triggered hydrogels are synthesized using pectin and PAM to facilitate colon-targeted delivery of budesonide for the treatment of UC. The polysaccharide, pectin, helps in colon targeting as it remains intact in the upper gastrointestinal tract and only degrades at the colon by colonic enzymes [23,24] while PAM acts as a pH-sensitive polymer which is insoluble at lower $\mathrm{pH}$ levels but becomes increasingly soluble as the $\mathrm{pH}$ increases [25]. The optimization of $\mathrm{pH}$-sensitive pectin/PAM hydrogel was carried out based on gel fraction, swelling behaviour, drug loading, and release. 


\section{Materials and Methods}

\subsection{Materials}

Acrylamide (AM), N,N'-methylene bisacrylamide (MBA), Potassium persulfate (KPS), and Pectin (PC) were purchased from Sigma-Aldrich. Sodium hydroxide, hydrochloric acid, and other reagents used in this study were of analytical grade and used without further purification.

\subsection{Methods}

\subsubsection{Preparation of Pectin/Polyacrylamide Hydrogel}

The hydrogels were prepared using acrylamide (AM) and pectin (PC) with the aid of an initiator and a crosslinker. Different amounts of AM were dissolved in $20 \mathrm{~mL}$ of PC solution followed by the addition of potassium persulfate (initiator) and MBA (crosslinker). The resultant mixture was placed in the oven at $60^{\circ} \mathrm{C}$ to allow the formation of hydrogel. The prepared hydrogels were immersed in distilled water for $120 \mathrm{~h}$ to remove any excess reactants. The hydrogels were dried in the oven at $60{ }^{\circ} \mathrm{C}$ for $24 \mathrm{~h}$.

\subsubsection{Determination of Gel Fraction}

The freshly prepared hydrogels were cut into discs using a mould of $1.0 \mathrm{~cm}$ diameter. The discs were placed in the oven at $60{ }^{\circ} \mathrm{C}$ to obtain a constant weight (G0). The dried discs were soaked in distilled water at room temperature for 7 days to extract any remaining AM or PC. The extracted discs were re-dried in an oven at $60^{\circ} \mathrm{C}$ to obtain a constant weight (G1). The percentage of gel fraction (\% GF) was calculated using the equation below:

$$
\% G F=(G 1 \mid G 0) \times 100
$$

\subsubsection{FT-IR Spectroscopic Analysis}

The IR spectra of samples were recorded using Perkin Elmer FT-IR Spectra 2000 at room temperature. KBr-disk method was used to prepare the samples. The samples were investigated over the range of $4000-400 \mathrm{~cm}^{-1}$.

\subsubsection{X-ray Diffraction}

X-ray diffractogram of the hydrogel was characterized by X-ray diffractometer (DBAdvance, Bruker) using CuKá radiation at $40 \mathrm{kV}$ and $50 \mathrm{~mA}$ in differential angle range of $5-70^{\circ} 2 \theta$.

\subsubsection{Differential Scanning Calorimetry (DSC)}

DSC thermogram of hydrogel was recorded using differential scanning calorimeter (Melter Toledo DSC Stare). About 3-4 mg of each sample was placed in the pan and heated over the temperature range of $20-200{ }^{\circ} \mathrm{C}$ with a heating rate of $10^{\circ} \mathrm{C} / \mathrm{min}$.

\subsubsection{Morphological Analysis}

Morphology of hydrogels were studied at different $\mathrm{pH}$. In this regard, dried hydrogel disk (weight approx. $100 \mathrm{mg}$ ) were soaked in various phosphate buffer $\mathrm{pHs}(1.5,5.8$, and 7.4) for $24 \mathrm{~h}$. Afterward hydrogel disks were washed with water and freeze-dried for morphology analysis. The external morphology and porous structure of the hydrogels were analysed using scanning electron microscopy (SEM) (SC500; BioRad, London, UK). The samples were mounted on an aluminum stub and coated with gold in a sputter coater under an argon atmosphere.

\subsubsection{Swelling Studies}

The swelling behaviour of the hydrogels was studied under various phosphate buffer pHs (1.5, 5.8, and 7.4). The dried extracted hydrogel disk (Gd) (approx. weight $100 \mathrm{mg}$ ) was soaked in $50 \mathrm{~mL}$ of media. At fixed time intervals $(30 \mathrm{~min}, 1 \mathrm{~h}, 2 \mathrm{~h}, 4 \mathrm{~h}, 6 \mathrm{~h}, 8 \mathrm{~h}$, and 
$24 \mathrm{~h}$ ), the weight of the swollen hydrogel was recorded (Gs). The study was conducted in triplicates. The percentage swelling $(\% S R)$ was calculated using the equation below:

$$
\% S R=(G s-G d \mid G d) \times 100
$$

\subsubsection{Drug Loading}

The dried hydrogels disk (weight $100 \mathrm{mg}$ ) was loaded with budesonide by placing the hydrogel disk (approx. $100 \mathrm{mg}$ ) in $5 \mathrm{~mL}$ of drug solution $(10 \mathrm{mg}$ ) in alcohol for $8 \mathrm{~h}$. The swollen hydrogels were removed and dried to a constant weight. The absorbance of the remaining solution was measured using a spectrophotometer and the concentration of the drug remaining was determined. Drug loading $(D L)$ and drug entrapment efficiency $(E E)$ were calculated using the equations below:

$$
\begin{gathered}
E E=(W o-W f \mid W o) \times 100 \\
D L=(W d g \mid W g) \times 100 .
\end{gathered}
$$

where $W_{0}$ is total amount of drug in the solution before loading, $W f$ is total amount of drug in the solution after loading, $W d g$ is the amount of drug incorporated into the hydrogel, and $W g$ is the weight of the dried hydrogel.

\subsubsection{In-Vitro Drug Release Study}

The drug-loaded hydrogels disk (approx. weight $108 \mathrm{mg}$ ) was immersed in $50 \mathrm{~mL}$ hydrochloric acid buffer $\mathrm{pH} 1.5$ for $2 \mathrm{~h}$. Then, the hydrogels were immersed in phosphate buffer $\mathrm{pH} 7.4$ for $48 \mathrm{~h}$. The drug-loaded hydrogels were also immersed in buffer $\mathrm{pH} 7.4$ alone to compare drug release profiles between the two systems. The dissolution media was kept at $37^{\circ} \mathrm{C}$ with constant agitation (50 rpm). At fixed time intervals, aliquots were withdrawn and substituted with fresh media. The concentration of the drug was measured at $272 \mathrm{~nm}$ using the spectrophotometer method. The results are demonstrated as percentage release over time.

\section{Results and Discussion}

\subsection{Synthesis of Hydrogels}

The $\mathrm{pH}$-sensitive PC/PAM hydrogel was prepared by grafting AM on PC with the aid of the initiator potassium persulfate followed by cross-linking with MBA. The reaction begins with the generation of sulfate ion radicals $\left(\mathrm{SO}^{4-}\right)$ from the initiator, potassium persulfate, upon conventional heating at $60^{\circ} \mathrm{C}$. In the presence of heat, these radicals react with water molecules to produce hydroxyl free radicals which were then used for the initiation of the polymerization process. The hydroxyl free radicals act on PC and AM and generate active sides on both the reactants. As a result, the reactants bind to form long chains of grafted polymers. Finally, to crosslink the polymer chains formed, MBA was added (Figure 1). Appearance of hydrogel was visual by Figure S1.

\subsubsection{Optimization of Pectin/Acrylamide Ratio}

To optimize the PC to AM ratio, various concentrations of PC and AM were added into the formulations while keeping the ratio of MBA to KPS constant (Table 1) and the ratio was optimized on the basis of gel fraction and percentage swelling. The hydrogel gel fraction (GF) represents the degree of cross-linking of the grafted polymer. Cross-linking ensures that the hydrogel does not dissolve and only expands in aqueous media. A greater degree of cross-linking results in higher GF. The results of the corresponding gel fraction of hydrogels (\%) are shown in Table 1. 


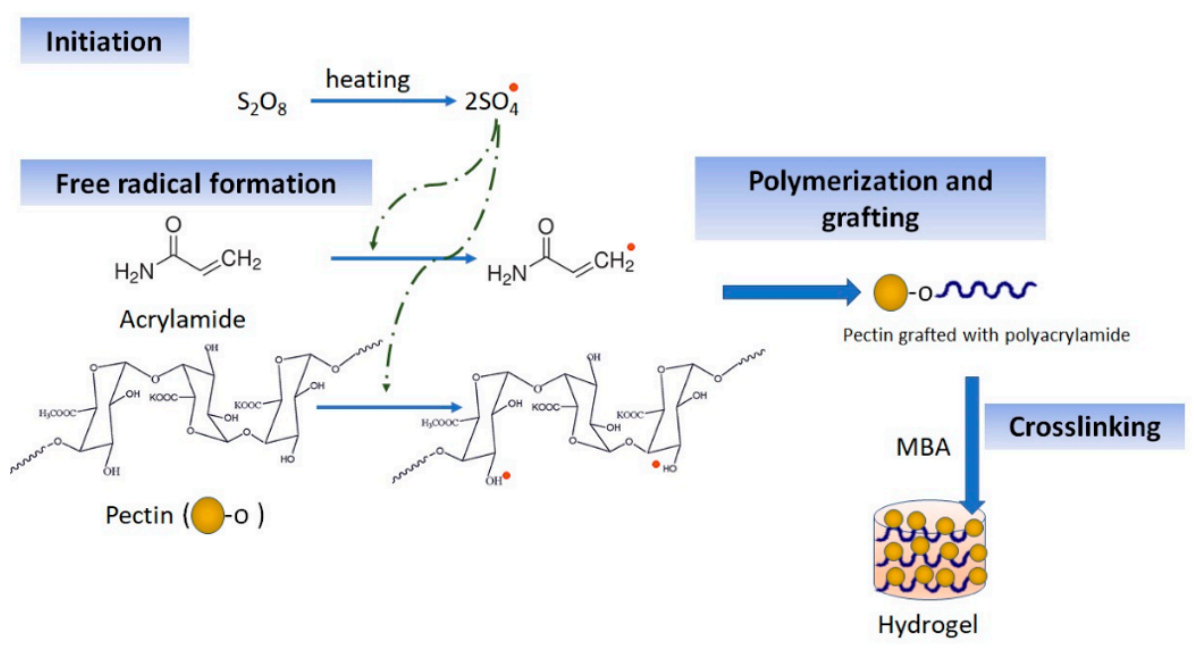

Figure 1. Reaction mechanism of hydrogel.

Table 1. Gel fraction of PC/polyacrylamide hydrogel based on different amounts of acrylamide and PC with a constant ratio of MBA/KPS.

\begin{tabular}{cccccc}
\hline $\begin{array}{c}\text { Formulation Code for } \\
\text { Hydrogel }\end{array}$ & $\begin{array}{c}\text { AM } \\
\mathbf{( g )}\end{array}$ & $\begin{array}{c}\text { PC } \\
\mathbf{( g )}\end{array}$ & $\begin{array}{c}\text { Ratio of } \\
\text { MBA/KPS }\end{array}$ & $\begin{array}{c}\text { Gel Fraction of } \\
\text { Hydrogel (\%) }\end{array}$ & SD \\
\hline 1A & 1.5 & 0.5 & $50 / 50$ & 58.9 & 0.63 \\
1B & 1.5 & 1 & $50 / 50$ & 49.01 & 2.17 \\
F3 & 2.1 & 0.5 & $50 / 50$ & 77.23 & 1.57 \\
1C & 2.1 & 1 & $50 / 50$ & 72.29 & 0.53 \\
1D & 2.8 & 0.5 & $50 / 50$ & 81.08 & 2.47 \\
1E & 2.8 & 1 & $50 / 50$ & 76.09 & 1.25 \\
\hline
\end{tabular}

As observed from Table 1, GF increased with an increase in the amount of AM (1.5-2.8 g) which contribute to higher grafting and polymerization at higher concentrations of AM. Yin et al. reported similar findings where poly(acrylic acid-co acrylamide)/carboxymethyl chitosan hydrogels were developed, and gel fraction was found leading to poly(acrylic acid-co acrylamide) in the reaction mixture [26]. Conversely, when the amount of PC was increased from $0.5 \mathrm{~g}$ to $1 \mathrm{~g}$, formulations of gel fraction deceased, as evident with $0.5 \mathrm{~g}$ PC yielding higher GF, as demonstrated in formulations $1 \mathrm{~A}(58.90 \%)$, F3 $(77.23 \%)$, and 1D (81.08\%) compared to formulation contains $1 \mathrm{~g}$ PC. GF of the hydrogel is dependent on both AM and PC concentrations. The highest GF was recorded with $1 \mathrm{D}$ formulation. However, this was not statistically different from F3 GF.

Secondly, swelling ratio (SR) was estimated for optimization of hydrogels which represent its water holding capacity. It is the key factor for drug loading and release. The super-absorbency of the PC/PAM hydrogel is due to ionic carboxylate and nonionic carboxamide functional groups. The presence of ionic groups in polymer chains increase SR because these ions are more toughly solvated rather than non-ionic groups in the aqueous media. The $\mathrm{pH}$-sensitivity of hydrogels was studied in phosphate buffer solutions ( $\mathrm{pH} 5.8$ and 7.4) and also using hydrochloric acid ( $\mathrm{pH}$ 1.5). As seen in Figure 2, the swelling behaviour of hydrogels indicated $\mathrm{pH}$-dependent swelling. The swelling percentage increased from $\mathrm{pH} 1.5$ to 7.4 with maximum swelling at $\mathrm{pH} 7.4$. However, there was no drastic increment from pH 5.8 to 7.4. This pattern could be due to the presence of carboxylate ions in the PAM chain. At $\mathrm{pH}<4.7$, the carboxylate group is protonated, whereas at $\mathrm{pH} 5-7$, deprotonation happens, creating an electrostatic repulsive force and instigating greater swelling. Additionally, the increment of AM concentration in hydrogels leads to a lower percentage of swelling, this may contribute to higher polymerization and 
crosslinking density. From the GF and SR results, formulation 3 (F3) was determined to be the optimised formulation.

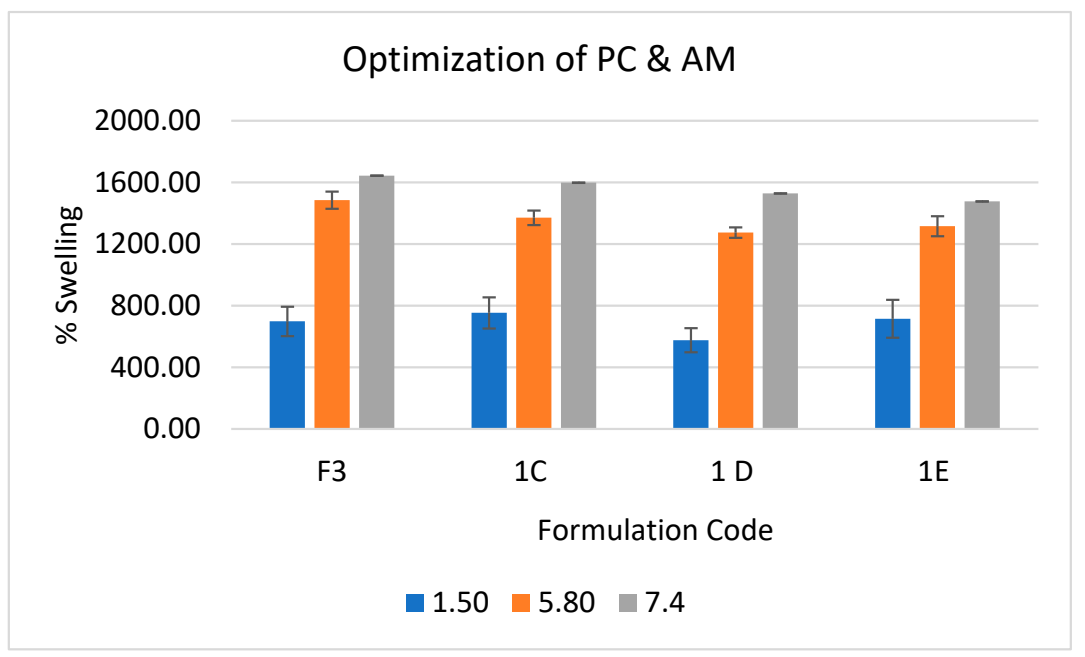

Figure 2. Swelling behaviour of hydrogels containing different PC/AM ratio at various phosphate buffer $\mathrm{pH}(1.5,5.8,7.4)$.

\subsubsection{Optimization of MBA/KPS Ratio}

To optimize the MBA to potassium persulfate ratio based on GF and SR, various concentrations of MBA and KPS were added into the formulations while keeping the ratio of AM to PC constant. The results of the corresponding gel fraction of hydrogel (\%) are shown in Table 2. It was observed that by increasing the concentration of MBA (F1-F5), the $\%$ gel fraction increased from $60.23 \%$ to $79.47 \%$. This suggests that the greater the amount of crosslinker added to the initial solution, the more cross-linked hydrogels will be formed, and hence a higher gel fraction of hydrogel. Similarly, the \% gel fraction of hydrogel was found to be greater with increasing concentration of potassium persulfate. For instance, it can be seen that formulation 7 with the highest amount of potassium persulfate has a gel fraction of $79.13 \%$ which is significantly different from formulation 6 $(63.4 \%)$ having the lowest amount of KPS. However, an insignificant difference was found when the concentration increased from $20 \mathrm{mg}$ to $50 \mathrm{mg}$ in $20 \mathrm{~mL}$ of reaction mixture.

Table 2. Gel fraction of PC/polyacrylamide hydrogel based on different amounts of initiator and cross linker with constant ratio of AM/PC.

\begin{tabular}{cccccc}
\hline $\begin{array}{c}\text { Formulation Code for } \\
\text { Hydrogel }\end{array}$ & $\begin{array}{c}\text { Ratio of } \\
\text { AM/PC }\end{array}$ & $\begin{array}{c}\text { MBA } \\
\text { (mg) }\end{array}$ & KPS (mg) & $\begin{array}{c}\text { Gel Fraction of } \\
\text { Hydrogel (\%) }\end{array}$ & SD \\
\hline F1 & $2.1 / 0.5$ & 10 & 50 & 60.23 & 1.03 \\
F2 & $2.1 / 0.5$ & 20 & 50 & 62.81 & 0.98 \\
F3 & $2.1 / 0.5$ & 50 & 50 & 77.23 & 1.57 \\
F4 & $2.1 / 0.5$ & 70 & 50 & 78.05 & 0.86 \\
F5 & $2.1 / 0.5$ & 90 & 50 & 79.47 & 1.73 \\
F6 & $2.1 / 0.5$ & 50 & 20 & 63.4 & 0.78 \\
F7 & $2.1 / 0.5$ & 50 & 70 & 79.13 & 1.18 \\
\hline
\end{tabular}

It is assumed that when the initiator concentration is increased, more radicals are produced from the initiator and utilised to produce growing monomer radicals. This eventually results in the maximum gel fraction of hydrogel, and at some point, saturation occurred that led to an insignificant increment in GF. Additionally, Figure 3 showed a lower SR value with increment in MBA and KPS concentration due to high generation of free radical and high crosslinking which restrict the swelling of the hydrogel. The $\%$ gel fraction 
and SR data pointed towards formulation 3 as the optimized formulation due to the high gel fraction and small statistical difference.

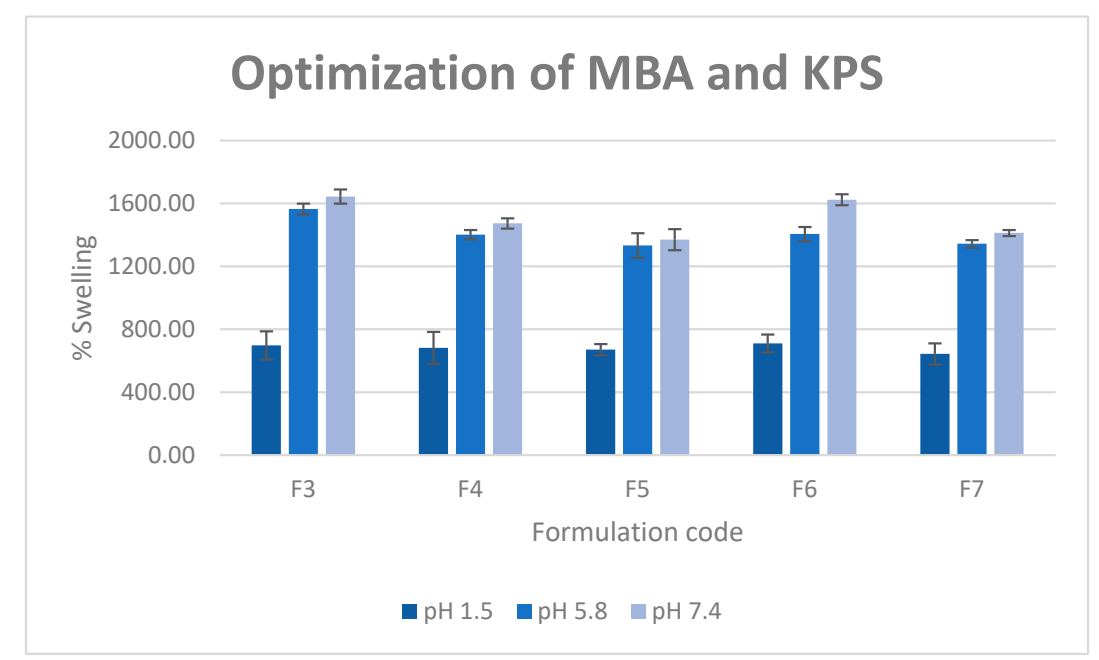

Figure 3. Swelling behaviour of hydrogels contains different amount of MBA and KPS at various phosphate buffer $\mathrm{pH}(1.5,5.8,7.4)$.

\subsection{Characterization of Budesonide Loaded Optimized Hydrogel}

After optimization of polymer, crosslinker, and initiator, optimized formulation (F3) was loaded with budesonide and characterized on the different parameters to evaluate compatibility, surface morphology, loading and release.

\subsubsection{FT-IR Spectroscopic Analysis}

Figure 4 shows the FTIR spectra of pure PC, PAM, F3 without budesonide, F3 with budesonide and budesonide. The spectrum of pure PC showed an absorption peak at $3568 \mathrm{~cm}^{-1}$ indicating the stretching of $-\mathrm{OH}$ groups. The peak observed at $2935 \mathrm{~cm}^{-1}$ is attributed to $-\mathrm{C}-\mathrm{H}$ stretching vibration. The peak at $1750 \mathrm{~cm}^{-1}$ represents $>\mathrm{C}=\mathrm{O}$ stretching vibrations on account of the presence of $-\mathrm{COOCH} 3$ group. The peaks at $1458 \mathrm{~cm}^{-1}$ and $1419 \mathrm{~cm}^{-1}$ could be due to $-\mathrm{CH} 2$ vibration and $-\mathrm{OH}$ bending vibration, respectively. The presence of a $-\mathrm{CH}-\mathrm{OH}$ group in aliphatic cyclic secondary alcohol was shown by the peak at $1149 \mathrm{~cm}^{-1}$. Next, according to the spectra of hydrogel with and without drug, common peaks between 3209 and $3250 \mathrm{~cm}^{-1}$ were observed in hydrogels. These peaks suggested $-\mathrm{OH}$ and $-\mathrm{NH}$ stretching. It was also observed that these peaks are more intense in the spectra of F3 with the drug compared to the spectra of F3 without the drug. This is due to the presence of $-\mathrm{OH}$ groups in the structure of budesonide which indicates that the hydrogel has been loaded with the drug successfully. Next, the peak at $1650 \mathrm{~cm}^{-1}$, as seen in both hydrogel spectra, indicated amide-I band ( $\mathrm{C}=\mathrm{O}$ stretching vibration) of the amide group which is the characteristic group of PAM. Similarly, amide-II bands within the range of $1510-1533 \mathrm{~cm}^{-1}$ were due to $\mathrm{C}-\mathrm{N}$ stretching vibrations as well as $\mathrm{N}-\mathrm{H}$ bending. The presence of these amide groups in the spectra of the hydrogels indicates the formation of grafted polymer. The peak at $1751 \mathrm{~cm}^{-1}$ could be assigned to free carboxylic groups while the peak at $2935 \mathrm{~cm}^{-1}$ corresponds to $\mathrm{C}-\mathrm{H}$ stretching of-CH2 groups. Lastly, the peaks of $-\mathrm{CH}$ bending of $-\mathrm{CH} 2$ group lie at $1350-1460 \mathrm{~cm}^{-1}$.

\subsubsection{X-ray Diffraction}

From Figure 5, the $X$-ray diffractogram of pure PC shows two broad peaks at $2 \theta$ equals to $15.10^{\circ} \theta$ and $21.36^{\circ} \theta$, indicating the amorphous nature of PC. Broad peaks can also be observed from the X-ray diffractogram of PAM at $2 \theta$ equals to $16.02^{\circ} \theta, 21.50^{\circ} \theta, 27.42^{\circ} \theta$, $34.37^{\circ} \theta, 41.13^{\circ} \theta$, and $52.87^{\circ} \theta$, indicating the amorphous nature of PAM. As a result of both 
parent compounds being amorphous, the resultant grafted polymer (hydrogel) had an increased amorphous nature.

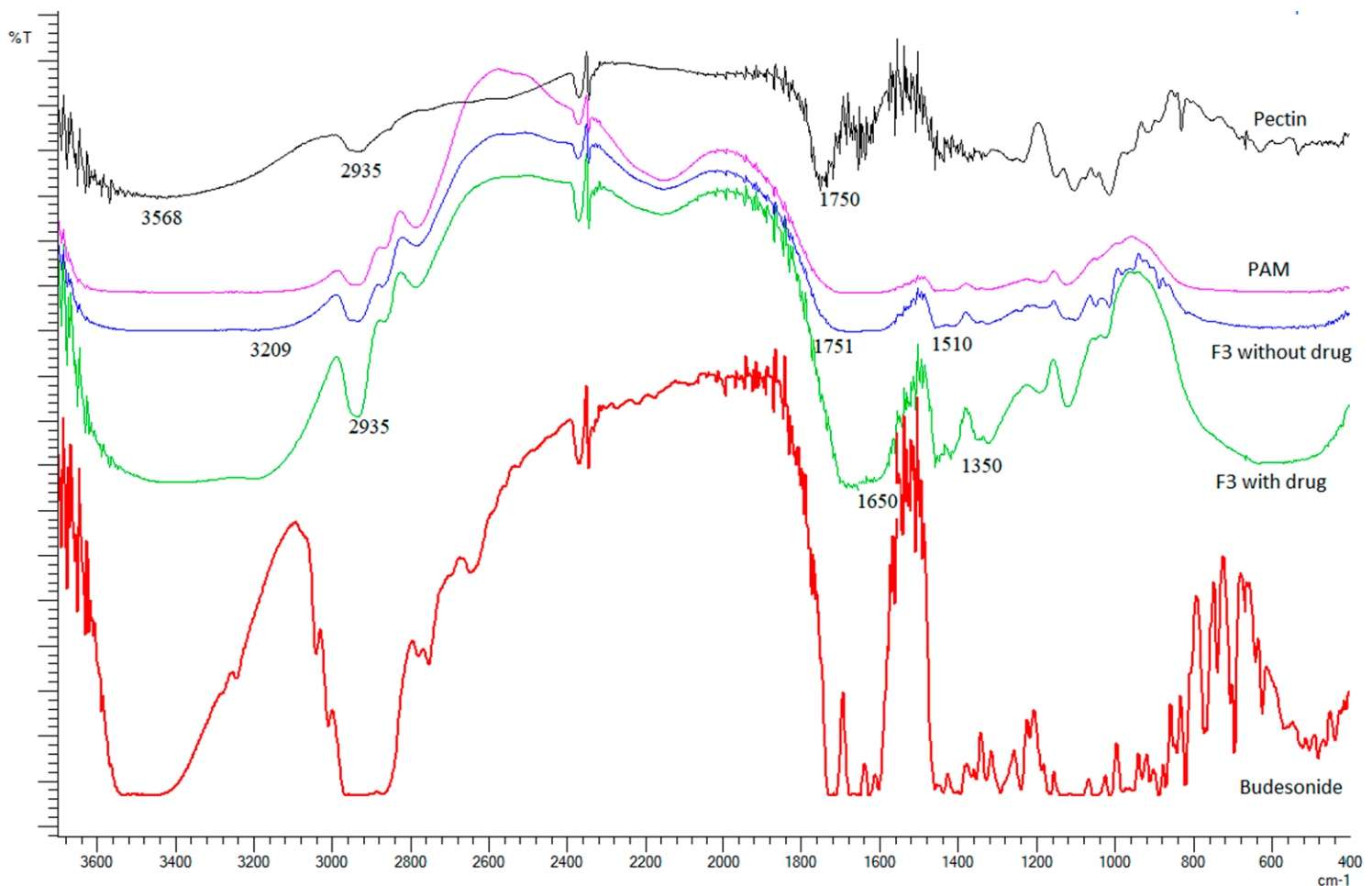

Figure 4. FTIR spectra of pure PC, polyacrylamide, F3 without drug, F3 with drug and budesonide.

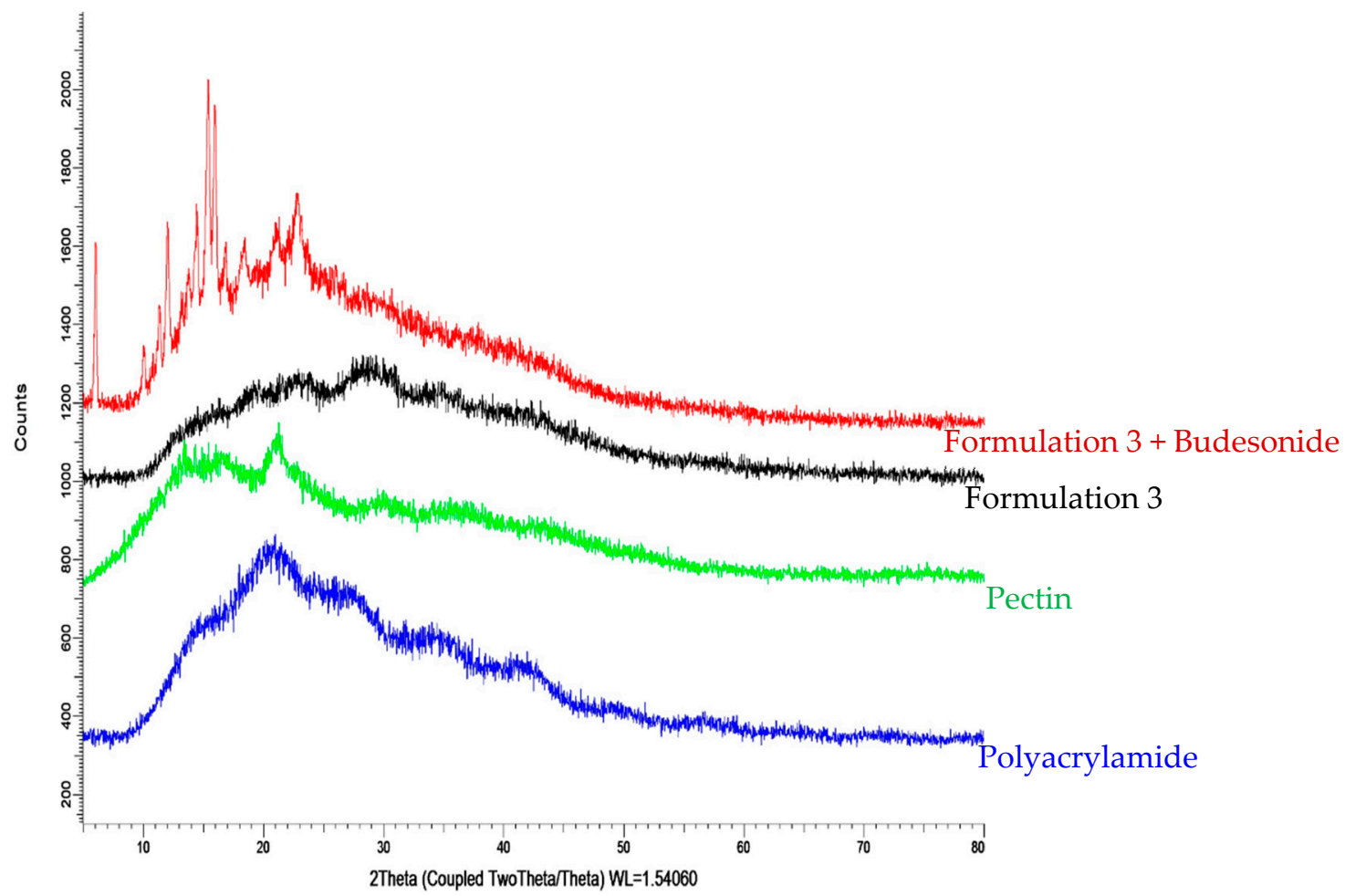

Figure 5. X-RD analysis of pectin, polyacrylamide (PAM), loaded hydrogel (Formulation $3+$ drug) and unloaded hydrogel (Formulation3). 
The X-ray diffractograms of the unloaded hydrogels show broad peaks between $150 \theta-40 \mathrm{o} \theta$, which can be attributed to the interactions between amorphous PC and PAM. Diffractogram of pure AM shows sharp crystalline peaks at $2 \theta$ equals to $12.02 \mathrm{o} \theta, 19.58 \mathrm{o} \theta$, $24.13 \mathrm{o} \theta, 28.69 \mathrm{o} \theta$, and $36.48 \mathrm{o} \theta$, which clearly indicate the crystalline behaviour of AM. As the hydrogels were highly amorphous and devoid of any sharp crystalline peaks, this indicates that AM was fully polymerized and properly extracted from the grafted polymer. Moreover, Figure 6 indicates crystalline nature of budesonide with many intense peaks in $X$-ray diffractogram between $10 \mathrm{o} \theta$ to $25 \mathrm{o} \theta$. There is an increase in the crystalline nature of the budesonide-loaded hydrogel (19.6\%) as compared to the unloaded hydrogel (10.6\%), due to the high crystallinity of budesonide. Characteristic peaks of budesonide can be seen in the X-ray diffractogram of the loaded hydrogel, indicating that the hydrogel has been loaded with the drug successfully.

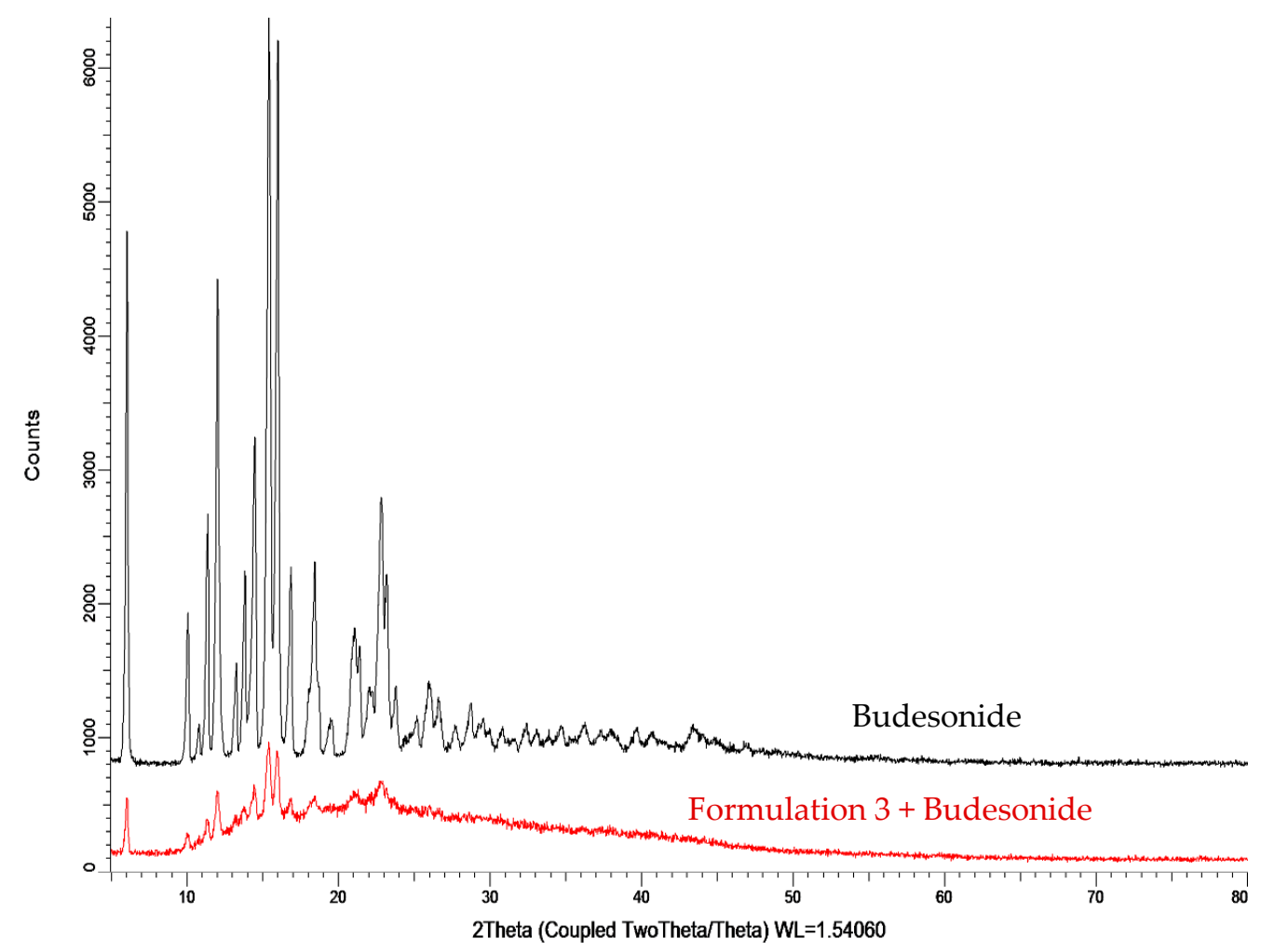

Figure 6. X-RD analysis budesonide and the loaded hydrogel.

\subsubsection{Differential Scanning Calorimetry (DSC)}

The DSC thermograms of PC and PC/PAM hydrogel (F3) are shown in Figure 7. In DSC of PC, a small peak at $74{ }^{\circ} \mathrm{C}$ was observed. The peak represents the glass transition temperature (Tg) of pure PC. On the other hand, the thermogram of hydrogel showed a peak at temperature $84{ }^{\circ} \mathrm{C}$ which corresponds to glass transition temperature $(\mathrm{Tg})$ of the grafted PC. This shows that there is an increase in Tg of the pure PC after blending with PAM. The increase in Tg may be attributed to the presence of intermolecular hydrogen bonding between PC $(-\mathrm{OH})$ and PAM $(-\mathrm{C}=\mathrm{O})$, indicating the formation of a grafted polymer. Similarly, the thermogram of PAM showed the occurrence of endothermic transitions around $170^{\circ} \mathrm{C}$. Budesonide did not show any sharp peak as stated in the literature. Therefore, further investigation is required in this context (Figure S2). 


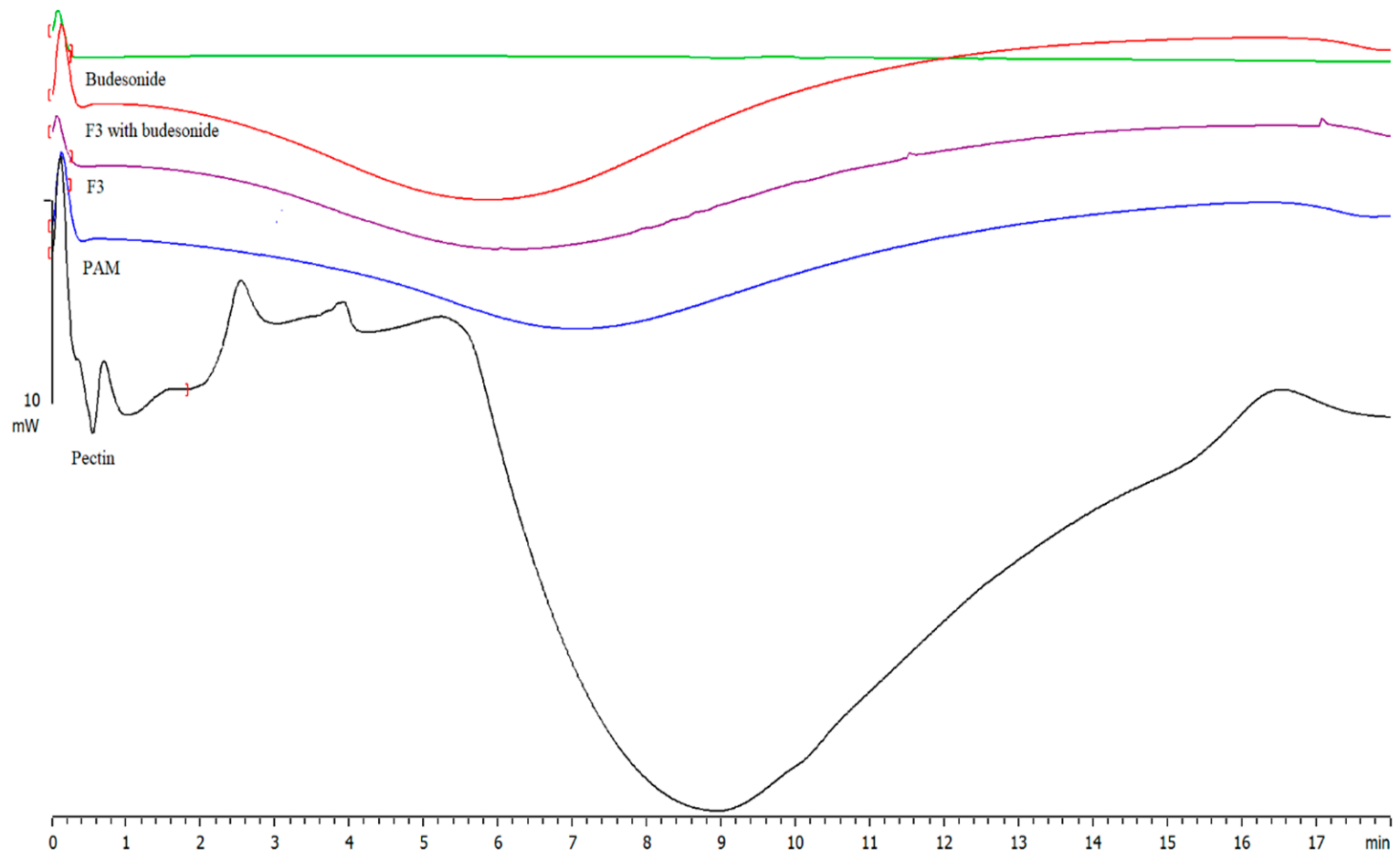

Figure 7. DSC thermogram of pure pectin, polyacrylamide, F3 without the drug, F3 with drug and budesonide.

\subsubsection{Morphological Analysis}

The surface morphology of budesonide hydrogel was investigated by scanning electron microscope at $300 \times$ magnification and it was revealed from Figure 8 the surface of hydrogels has interconnected pores at $\mathrm{pH} 5.8$ and $\mathrm{pH} 7.4$, however no pores appeared at acidic $\mathrm{pH}$, which supports the $\mathrm{pH}$-sensitive nature of the hydrogel. Prior studies have reported that interconnected pores and spongy structures allow greater diffusion of solvent molecules during swelling. The presence of a continuous porosity network would also enhance the water sorption capacity of the hydrogel [27]. The porosity plays multiple roles of enhancing the total water sorption capability and the rate of response by reducing the transport resistance. Therefore, the creation of porosity in hydrogels has been considered an important process in many ways [28].

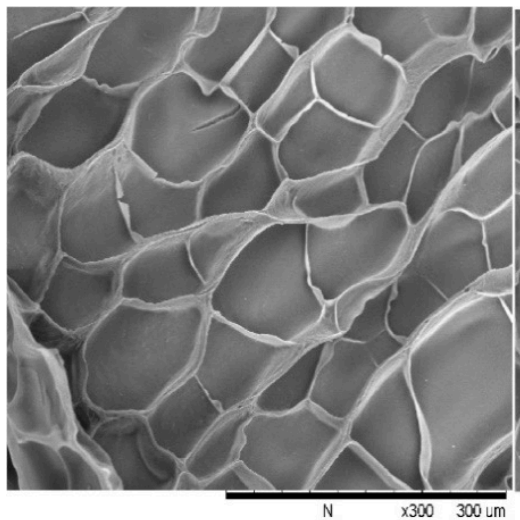

Swelling at pH 1.5

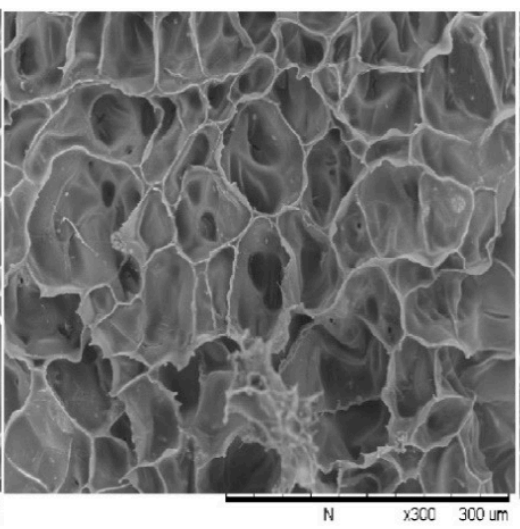

pH 5.8

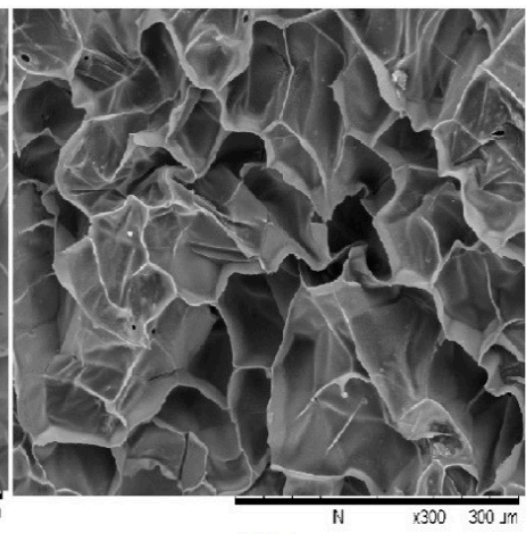

pH 7.4

Figure 8. SEM images of the hydrogel at different $\mathrm{pH}(1.5,5.8,7.4)$ at magnification $300 \times$. 


\subsubsection{Drug Loading and In-Vitro Drug Release}

On the basis of gel fraction and swelling, hydrogel-F3 was selected for drug loading (DL) and release. The EE of F3 was $80.4 \pm 3.34 \%$ respectively, and the DL was $8.89 \pm 1.35 \%$, respectively. The in vitro release profile of hydrogel principally depends on percentage swelling of hydrogel in dissolution media, solubility of the drug in media, and interaction of the drug with the polymeric network [29]. The release pattern was estimated in phosphate buffer at hydrochloric acid buffer $\mathrm{pH} 1.5$ for $2 \mathrm{~h}$ followed by $\mathrm{pH}$ 7.4. The cumulative percentage release in phosphate buffer $\mathrm{pH} 1.5$ during the first $2 \mathrm{~h}$ in $\mathrm{F} 3$ was $7 \%$, while the cumulative release in $\mathrm{pH} 7.4$ for next $3 \mathrm{~h}$ was $36 \%$. The same pattern was observed in total cumulative release (Figure 9), attributable to the swelling behaviour of the hydrogel with varying $\mathrm{pH}$. The drug release pattern was characterised by a biphasic, burst release, resulting from the presence of the drug on the hydrogel surface, followed by sustained release. Initially, the rapid release was observed because the concentration gradient functioned as the driving force for release, followed by a reduced release rate due to the depletion of the drug near the surface. According to Graham and McNeil (1984), hydrogel combines two components, one being a strong polymer network and the other being permeation channels filled with water for the diffusion of water-soluble solute. Thus, the release of the hydrogel mainly governs by the water content of the hydrogel. So, the hydrogel with greater water content is expected to release a higher amount of solute.

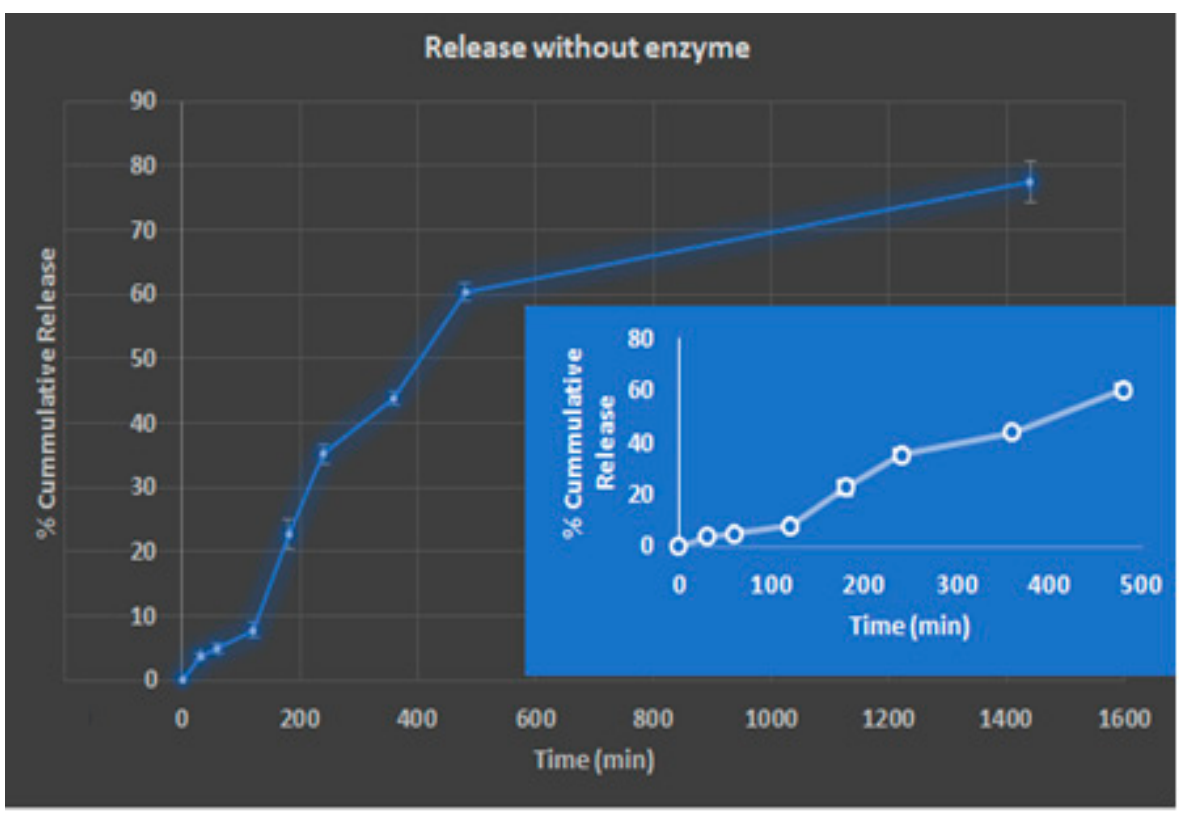

Figure 9. Graph of cumulative percentage drug release over time.

The correlation coefficient $\left(R^{2}\right)$ of $F 3$ was found to be $0.9772,0.8720,0.9820$, and 0.9390 for zero order, first order, Higuchi, and Korsmeyer-Peppas respectively (Table 3). The data revealed that release pattern of hydrogel was best fitted into zero-order kinetics as confirmed by higher $\mathrm{R}^{2}$. This denotes the constant release of drug from hydrogel for stipulated time. Additionally, Higuchi was used to identify the release mechanism and represent diffusion as the dominant release mechanism. As discussed earlier, at basic $\mathrm{pH}$, porous channels formed in hydrogel which leads to the slow diffusion of drug from formulation. Furthermore, the value of the release exponent of Korsmeyer-Peppas (n) in F3 was 0.697 , which is between 0.45 and 0.89 , which represents the non-Fickian diffusion, indicating that release of the drug via a dominant swelling-controlled diffusion mechanism along with slight surface erosion that occurred concurrently. 
Table 3. Drug release kinetics of drug-loaded hydrogel.

\begin{tabular}{cccccc}
\hline Formulation & $\begin{array}{c}\text { Zero-Order } \\
\left(\mathbf{R}^{\mathbf{2}}\right)\end{array}$ & $\begin{array}{c}\text { First-Order } \\
\mathbf{( R}^{\mathbf{2}}\end{array}$ & $\begin{array}{c}\text { Higuchi } \\
\mathbf{( R}^{\mathbf{2}}\end{array}$ & $\begin{array}{c}\text { Hixon-Crowell } \\
\mathbf{( R}^{\mathbf{2}}\end{array}$ & $\begin{array}{c}\text { Korsmeyer- } \\
\text { Peppas (n) }\end{array}$ \\
\hline $\begin{array}{c}\text { Drug loaded } \\
\text { hydrogel (F3) }\end{array}$ & 0.9772 & 0.8720 & 0.9320 & 0.9390 & 0.697 \\
\hline
\end{tabular}

\section{Conclusions}

In this study, we have reported the development and optimization of $\mathrm{pH}$-sensitive PC/PAM hydrogel for budesonide by grafting AM on PC with the aid of the initiator potassium persulfate followed by cross-linking with MBA. XRD studies showed the amorphous nature of hydrogel and loading of drug in hydrogel. Moreover, an increase in the crystallinity of the budesonide-loaded hydrogel was observed compared to the unloaded hydrogel, due to the high crystallinity of budesonide. DSC thermograms illustrate the increase in $\mathrm{Tg}$ of grafted polymer which is attributed to the presence of an intermolecular hydrogen bond. Additionally, the hydrogel revealed $\mathrm{pH}$-dependent swelling and biphasic release of the drug from the hydrogel. This release pattern showed the burst release of budesonide from the surface followed by sustain release with non-ficken diffusion mechanism. Therefore, PC/PAM hydrogel could be a promising approach for the colon-targeted delivery of budesonide for the treatment of ulcerative colitis. Future scopes of investigation include cytotoxicity and stability studies, along with in-vivo pre-clinical effectiveness.

Supplementary Materials: The following are available online, Figure S1: Photos of prepared hydrogels. Figure S2: DSC thermogram of budesonide; budesonide loaded hydrogel; polyacrylamide.

Author Contributions: Conceptualization, M.P., M.C.I.M.A., and H.C.; methodology, M.P., M.C.I.M.A., and H.C.; formal analysis M.P., and H.C.; resources, M.P., BG, SKB and H.C.; data curation, S.K.D.S.S. and N.C.A.; writing - original draft preparation, S.K.D.S.S. and N.C.A.; writing-review and editing SKB; BG; M.P., M.C.I.M.A., and H.C.; project administration, M.P. and H.C.; funding acquisition, M.P. and H.C. All authors have read and agreed to the published version of the manuscript.

Funding: This research was funded by International Medical University, grant number BP I01/2018(17) and the APC was funded by International Medical University.

Institutional Review Board Statement: The study was approved by the Institutional Review Board of International Medical University (protocol code BP I-01/2018(17) and May 2018).

Informed Consent Statement: Not applicable.

Data Availability Statement: Data is freely available.

Conflicts of Interest: The authors declare no conflict of interest.

Sample availability: The data presented in this study are available on request from the corresponding author.

\section{References}

1. Danese, S.; Fiocchi, C. Ulcerative Colitis. N. Engl. J. Med. 2011, 365, 1713-1725. [CrossRef] [PubMed]

2. Ungaro, R.; Mehandru, S.; Allen, P.B.; Peyrin-Biroulet, L.; Colombel, J.-F. Ulcerative colitis. Lancet 2017, 389, 1756-1770. [CrossRef]

3. Bressler, B.; Marshall, J.K.; Bernstein, C.N.; Bitton, A.; Jones, J.; Leontiadis, G.I.; Panaccione, R.; Steinhart, A.H.; Tse, F.; Feagan, B.; et al. Clinical practice guidelines for the medical management of nonhospitalized ulcerative colitis: The Toronto consensus. Gastroenterology 2015, 148, 1035-1058. [CrossRef]

4. Kornbluth, A.; Sachar, D. Erratum: Ulcerative Colitis Practice Guidelines in Adults: American College of Gastroenterology, Practice Parameters Committee. Am. J. Gastroenterol. 2010, 105, 500. [CrossRef]

5. Seibold, F.; Fournier, N.; Beglinger, C.; Mottet, C.; Pittet, V.; Rogler, G.; Swiss IBD Cohort Study Group. Topical therapy is underused in patients with ulcerative colitis. J. Crohn's Colitis 2014, 8, 56-63. [CrossRef] [PubMed]

6. Abdalla, M.I.; Herfarth, H. Budesonide for the treatment of ulcerative colitis. Expert Opin. Pharmacother. 2016, 17, 1549-1559. [CrossRef] [PubMed] 
7. Danese, S.; Hart, A.; Dignass, A.; Louis, E.; D’Haens, G.; Dotan, I.; Rogler, G.; D’Agay, L.; Iannacone, C.; Peyrin-Biroulet, L. Effectiveness of budesonide MMX (Cortiment) for the treatment of mild-to-moderate active ulcerative colitis: Study protocol for a prospective multicentre observational cohort study. BMJ Open Gastroenterol. 2016, 3, e000092. [CrossRef] [PubMed]

8. Karolewska-Bochenek, K.; Dziekiewicz, M.; Banaszkiewicz, A. Budesonide MMX in Paediatric Patients with Ulcerative Colitis. J. Crohn's Colitis 2017, 11, 1402. [CrossRef]

9. Lautenschläger, C.; Schmidt, C.; Fischer, D.; Stallmach, A. Drug delivery strategies in the therapy of inflammatory bowel disease. Adv. Drug Deliv. Rev. 2014, 71, 58-76. [CrossRef]

10. Drug Approval Package (Budesonide). Available online: https://www.accessdata.fda.gov/drugsatfda_docs/nda/2006/021949_ budesonide_toc.cfm (accessed on 20 August 2020).

11. Sherlock, M.E.; MacDonald, J.K.; Griffiths, A.M.; Steinhart, A.H.; Seow, C.H. Oral budesonide for induction of remission in ulcerative colitis. Cochrane Database Syst. Rev. 2015. [CrossRef]

12. Sandborn, W.J.; Danese, S.; D’Haens, G.; Moro, L.; Jones, R.; Bagin, R.; Huang, M.; David Ballard, E.; Masure, J.; Travis, S. Induction of clinical and colonoscopic remission of mild-to-moderate ulcerative colitis with budesonide MMX 9 mg: Pooled analysis of two phase 3 studies. Aliment. Pharmacol. Ther. 2015, 41, 409-418. [CrossRef]

13. Lichtenstein, G.R. Budesonide Multi-matrix for the Treatment of Patients with Ulcerative Colitis. Dig. Dis. Sci. 2016, 61, 358-370. [CrossRef]

14. Yoo, J.-W.; Naeem, M.; Cao, J.; Choi, M.; Kim, W.; Moon, H.R.; Lee, B.L.; Kim, M.-S.; Jung, Y. Enhanced therapeutic efficacy of budesonide in experimental colitis with enzyme/pH dual-sensitive polymeric nanoparticles. Int. J. Nanomed. 2015, 10, 4565-4580. [CrossRef]

15. Zhang, L.; Sang, Y.; Feng, J.; Li, Z.; Zhao, A. Polysaccharide-based micro/nanocarriers for oral colon-targeted drug delivery. J. Drug Target. 2016, 24, 579-589. [CrossRef]

16. Chourasia, M.K.; Jain, S.K. Polysaccharides for Colon Targeted Drug Delivery. Drug Deliv. 2004, 11, 129-148. [CrossRef] [PubMed]

17. Chourasia, M.K.; Jain, S.K. Design and Development of Multiparticulate System for Targeted Drug Delivery to Colon. Drug Deliv. 2004, 11, 201-207. [CrossRef]

18. Philip, A.; Philip, B. Colon Targeted Drug Delivery Systems: A Review on Primary and Novel Approaches. Oman Med. J. 2010, 25, 70-78. [CrossRef] [PubMed]

19. Liu, L.; Fishman, M.; Kost, J.; Hicks, K. Pectin-based systems for colon-specific drug delivery via oral route. Biomaterials 2003, 24, 3333-3343. [CrossRef]

20. Liu, Y.; Zhou, H. Budesonide-Loaded Guar Gum Microspheres for Colon Delivery: Preparation, Characterization and In Vitro/In Vivo Evaluation. Int. J. Mol. Sci. 2015, 16, 2693-2704. [CrossRef] [PubMed]

21. Ahmed, E.M. Hydrogel: Preparation, characterization, and applications: A review. J. Adv. Res. 2015, 6, 105-121. [CrossRef] [PubMed]

22. Wen, H.; Jung, H.; Li, X. Drug Delivery Approaches in Addressing Clinical Pharmacology-Related Issues: Opportunities and Challenges. AAPS J. 2015, 17, 1327-1340. [CrossRef]

23. Zhang, W.; Mahuta, K.M.; Mikulski, B.A.; Harvestine, J.N.; Crouse, J.Z.; Lee, J.C.; Kaltchev, M.G.; Tritt, C.S. Novel pectin-based carriers for colonic drug delivery. Pharm. Dev. Technol. 2016, 21, 127-130. [CrossRef] [PubMed]

24. Varshosaz, J.; Emami, J.; Tavakoli, N.; Minaiyan, M.; Rahmani, N.; Dorkoosh, F.; Mahzouni, P. Pectin Film Coated Pellets for Colon-Targeted Delivery of Budesonide: In-Vitro/In-Vivo Evaluation in Induced Ulcerative Colitis in Rat. Iran. J. Pharm. Res. IJPR 2012, 11, 733-745.

25. Prasad, S.; Aeri, V. Approaches for Targeted Drug Delivery to Colon. Int. J. Drug Deliv. Technol. 2010, 3, 8-11. [CrossRef]

26. Yin, L.; Fei, L.; Cui, F.; Tang, C.; Yin, C. Superporous hydrogels containing poly(acrylic acid-co-acrylamide)/O-carboxymethyl chitosan interpenetrating polymer networks. Biomaterials 2007, 28, 1258-1266. [CrossRef]

27. Halib, N.; Amin, M.C.I.M.; Ahmad, I. Unique stimuli responsive characteristics of electron beam synthesized bacterial cellulose/acrylic acid composite. J. Appl. Polym. Sci. 2010, 116, 2920-2929. [CrossRef]

28. Sadeghi, M.; Heidari, B. Crosslinked Graft Copolymer of Methacrylic Acid and Gelatin as a Novel Hydrogel with pHResponsiveness Properties. Materials 2011, 4, 543-552. [CrossRef]

29. Rizwan, M.; Yahya, R.; Hassan, A.; Yar, M.; Azzahari, A.; Selvanathan, V.; Sonsudin, F.; Abouloula, C. Erratum: pH Sensitive Hydrogels in Drug Delivery: Brief History, Properties, Swelling, and Release Mechanism, Material Selection and Applications. Polymers 2017, 9, 137. Polymers 2017, 9, 225. [CrossRef] 